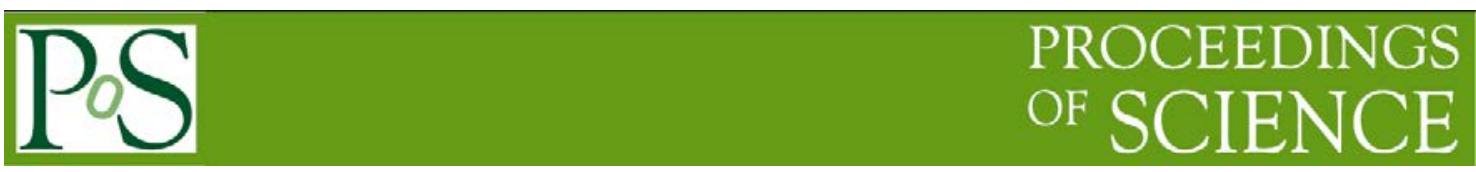

\title{
Dijet Imbalance in 2.76 TeV PbPb Collisions in CMS
}

\author{
David Krofcheck* \\ On behalf of the CMS Collaboration \\ Department of Physics \\ The University of Auckland \\ Private Bag 92010 \\ Auckland, New Zealand \\ E-mail:d.krofcheck@auckland.ac.nz
}

\begin{abstract}
We report on dijet production in $\mathrm{PbPb}$ collisions at a nucleon-nucleon center-of-mass energy of $2.76 \mathrm{TeV}$ studied with the CMS detector at the LHC. A large dijet momentum imbalance is observed in central $\mathrm{PbPb}$ collisions, which reduces in the more peripheral collisions. This observation is consistent with a jet quenching scenario, where the partons lose energy propagating through the hot and dense QCD medium. An initial study of $\gamma$-jet correlations is also presented.
\end{abstract}

36th International Conference on High Energy Physics

July 4-11, 2012

Melbourne, Australia

*Speaker 


\section{Introduction}

Ultrarelativistic nuclear collisions are used to study quantum chromodynamics (QCD) which is the fundamental theory of the strong interaction. Calculations in lattice QCD [1] lead to the conclusion that a state of deconfined quarks and gluons may be achieved at a critical temperature in the 150-175 MeV range. Such a hot and dense nuclear medium is investigated in this work through hadronic jet-jet and $\gamma$-jet correlations measured using the Compact Muon Solenoid (CMS) detector [2], and produced from $\sqrt{S_{N N}}=2.76 \mathrm{TeV} \mathrm{PbPb}$ collisions at the Large Hadron Collider (LHC).

High transverse momentum $\left(\mathrm{p}_{\mathrm{T}}\right)$ partons and photons can be produced in hard scatterings during the earliest times of a $\mathrm{PbPb}$ collision; e.g. $\tau \leq 0.1 \mathrm{fm} / \mathrm{c}$. In the absence of medium effects two jets will have equal magnitude $\mathrm{p}_{\mathrm{T}}$ and will be emitted back-to-back in the azimuthal direction. Due to collisions and medium induced gluon emission, the partons traveling through the medium lose energy resulting in a suppression of high- $\mathrm{p}_{\mathrm{T}}$ jets (called "jet quenching”) and a deviation from back-to-back alignment [3]. Figure 1 is illustrative of the difference in energy loss that is observed in dijet events in PbPb collisions at the LHC.

The amount of energy lost for each jet should depend upon the distance that a jet has traveled through the hot and dense matter region. Electroweak gauge bosons in coincidence with hadronic jets, $\mathrm{Z}^{0}$-jet and specifically in this work $\gamma$-jet, may be particularly useful in understanding hadronic energy loss $[4-5,8]$. The coincident $Z^{0} / \gamma$ will not suffer significant energy losses in the medium.

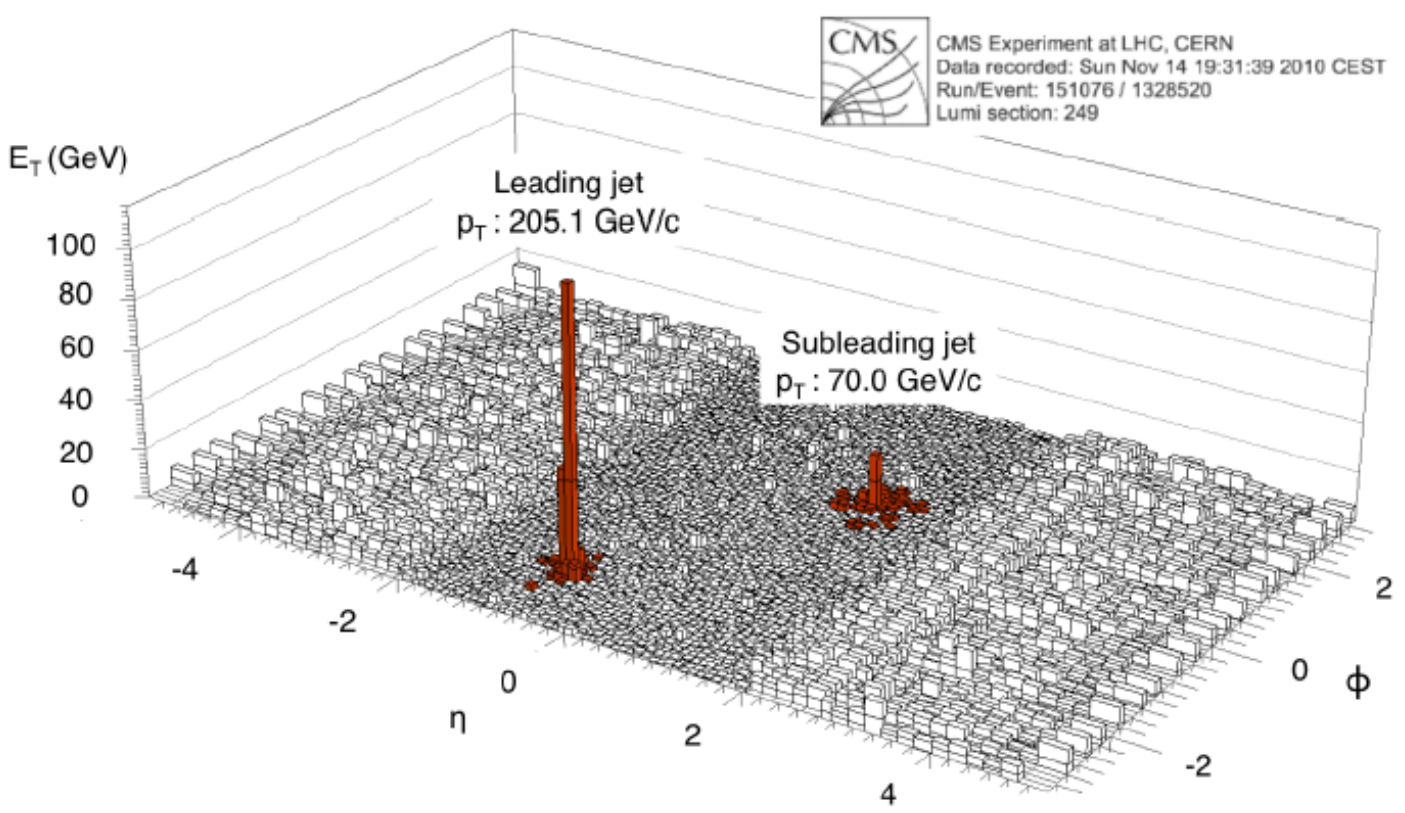

Figure 1: An imbalanced dijet event observed as the summed transverse energies of the CMS electromagnetic and hadronic calorimeters [6]. 


\section{Hadronic Dijet Imbalance in Pb-Pb Collisions}

A method to characterize the observed dijet transverse momentum imbalance is to define an asymmetry ratio:

$$
\mathrm{A}_{\mathrm{J}}=\frac{\mathrm{p}_{\mathrm{T}, 1}-\mathrm{p}_{\mathrm{T}, 2}}{\mathrm{p}_{\mathrm{T}, 1}+\mathrm{p}_{\mathrm{T}, 2}}
$$

In eq. 2.1 the higher energy ("leading”) jet is labelled $\mathrm{p}_{\mathrm{T}, 1}$ while the coincident lower energy ("sub leading") jet is labelled $\mathrm{p}_{\mathrm{T}, 2}$. The centrality dependence [7] of $\mathrm{A}_{\mathrm{J}}$ is shown in Figure 2, with the requirements: $\mathrm{p}_{\mathrm{T}, 1}>120 \mathrm{GeV} / \mathrm{c}$ and $\mathrm{p}_{\mathrm{T}, 2}>30 \mathrm{GeV} / \mathrm{c}$, with the angular separation $(\Delta \varphi)$ between the dijets chosen to be $\Delta \varphi>2 \pi / 3$. The hadronic jets are restricted to the CMS hadronic calorimeter pseudorapidity $(\eta)$ region of $|\eta|<2$. With $\eta=-\ln [\tan (\theta / 2)]$ and $\theta$ is the polar angle measured relative to the counterclockwise LHC Pb-ion beam. Jet reconstruction and appropriate background subtraction are described in [7].

The hardest (most central) collisions are illustrated in the bottom row of Figure 2 in which $\mathrm{A}_{\mathrm{J}}$ of the $\mathrm{PbPb}$ collision of $0-10 \%, 10-20 \%$ and $20-30 \%$ of the total inelastic $\mathrm{PbPb}$ cross section are measured. A larger imbalance is seen for these three most central collisions.

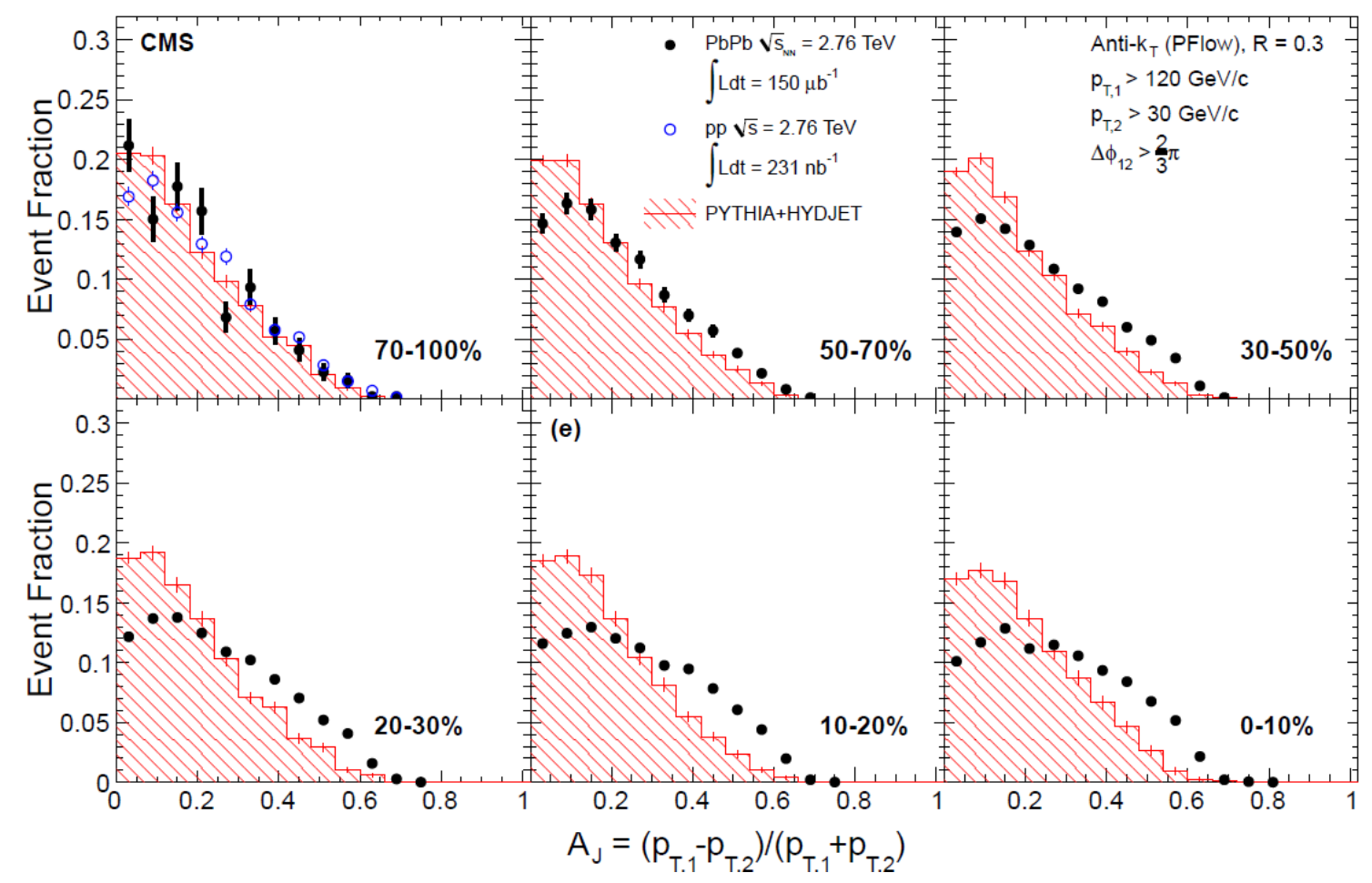

Figure 2: The centrality dependence of $A_{\mathrm{J}}$. Simulations using PYTHIA dijets embedded into HYDJET $\mathrm{PbPb}$ collisions are shown as the hatched areas. Superimposed upon the most peripheral collision data (70-100\% centrality bin) are data from pp collisions also at $2.76 \mathrm{TeV}$ [7]. 


\section{Hadronic jet $+\gamma$ Imbalance}

An observable quantity defined to examine the hadronic-jet $+\gamma$ transverse momentum imbalance is called the asymmetry fraction ratio $X_{J \gamma}=\mathrm{p}_{\mathrm{T}}^{\mathrm{Jet}} / \mathrm{p}_{\mathrm{T}}^{\gamma}$. So called "isolated-photons" were selected as described in [8]. In this analysis momentum cuts of $p_{T}^{\text {Jet }}>30 \mathrm{GeV} / \mathrm{c}$ and $\mathrm{p}_{\mathrm{T}}^{\gamma}>60 \mathrm{GeV} / \mathrm{c}$ are applied to the coincident hadronic jets and photons. The data presented in Figure 3 show the angular separation between jets and photons as a function of collision centrality [8] in the indicated $\eta$ regions. The photons were restricted to the electromagnetic calorimeter barrel region of $|\eta|<1.44$. In the Figure 3 data a cut is made on the hadronic jets, restricting them to $|\eta|<1.6$. The jet and photon reconstruction and identification are fully detailed in [8].

Plotted in Figure 3 are the number of jet + isolated $\gamma$ pairs $d_{J \gamma}$ per $d X_{J \gamma}$ interval, normalized by the total number of such pairs, as a function of $\mathrm{X}_{\mathrm{J} \gamma}$. The jets are observed to be well correlated in a direction directly opposite to the $\gamma$ ray direction $\left(\Delta \varphi_{\mathrm{J} \gamma} \approx \pi\right)$.

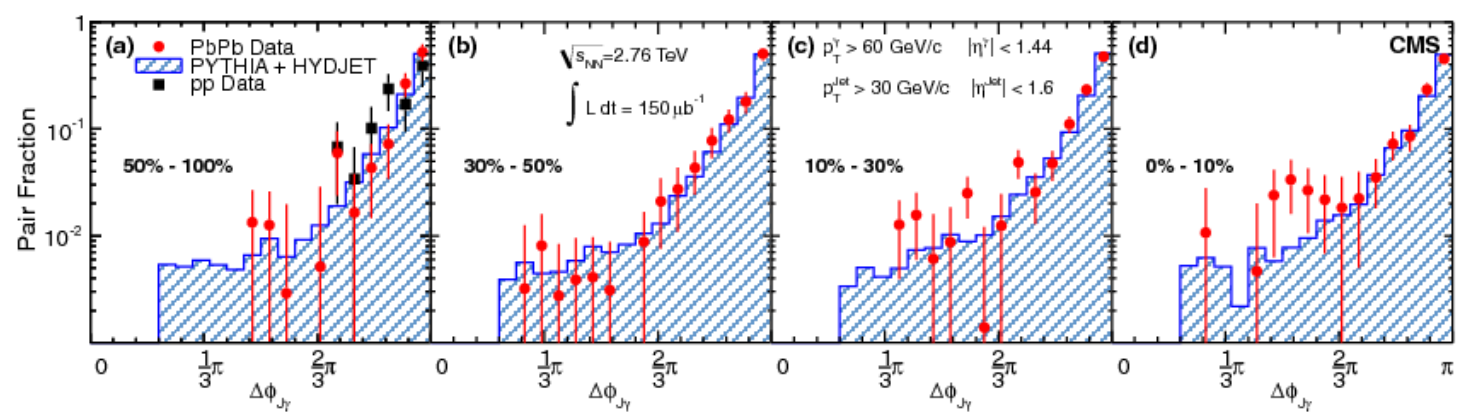

Figure 3: Angular correlations between jets and photons for different $\mathrm{PbPb}$ collision centrality bins [8]. Simulations using PYTHIA jet $+\gamma$ events are embedded into HYDJET PbPb collisions as shown as the hatched areas.

A tight cut is also placed upon $\Delta \varphi$ to suppress background jets. The data plotted in Figure 4 uses $\Delta \varphi>7 \pi / 8$ to examine the asymmetry ratio. This $\mathrm{PbPb}$ data exhibits a cutoff in $\mathrm{X}_{\mathrm{J \gamma}}$ due to the $30 \mathrm{GeV} / \mathrm{c}$ jet threshold. The simulated PYTHIA+HYDJET events show essentially no change in the peak location as the collision centrality changes.

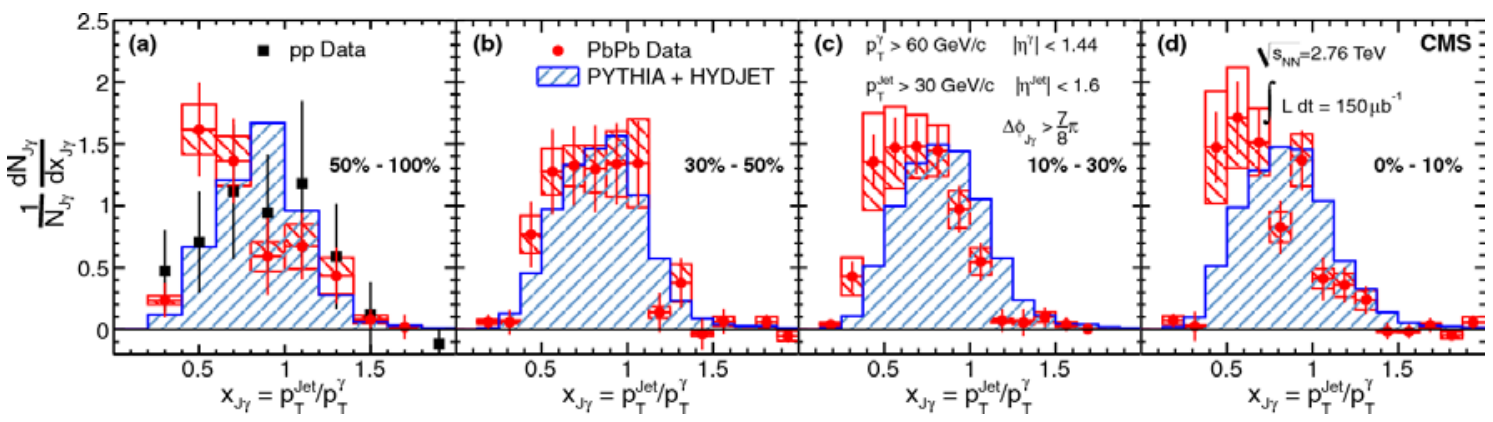

Figure 4: The centrality dependence of jet-to-photon transverse momentum. Simulations using PYTHIA jet $+\gamma$ events are embedded into HYDJET PbPb collisions as shown as the hatched areas. Data from 2.76 $\mathrm{TeV}$ pp collisions is shown superimposed on the results in the most peripheral bin (50-100\%) [8]. 
However, the data for $\mathrm{PbPb}$ central collisons show a larger deviation from the centroid of the PYTHIA+HYDJET simulations than do the data for more peripheral $\mathrm{PbPb}$ collisions.

The dependence of the average values $\left\langle\mathrm{X}_{\mathrm{J} \gamma}\right\rangle$ of the asymmetry frasction ratio as a function of participating nucleons $\left(\mathrm{N}_{\text {part }}\right)$ is presented in Figure 5(a). Medium effects are responsible for the greater jet $+\gamma$ imbalance when a larger number of nucleons participate in the $\mathrm{PbPb}$ collision. There are genuine jet $+\gamma$ events which do not contribute to $\left\langle\mathrm{X}_{J \gamma}\right\rangle$ because of the imposed 30 $\mathrm{GeV} / \mathrm{c}$ jet momentum threshold. To account for these events, in Figure 5(b) is shown the number of events ratio $R_{J \gamma}$ which is the fraction of isolated $\gamma^{\text {'s }}$ with its associated jet that fits both the tight $\Delta \varphi>7 \pi / 8$ angular cut and $30 \mathrm{GeV} / \mathrm{c}$ jet momentum cut.
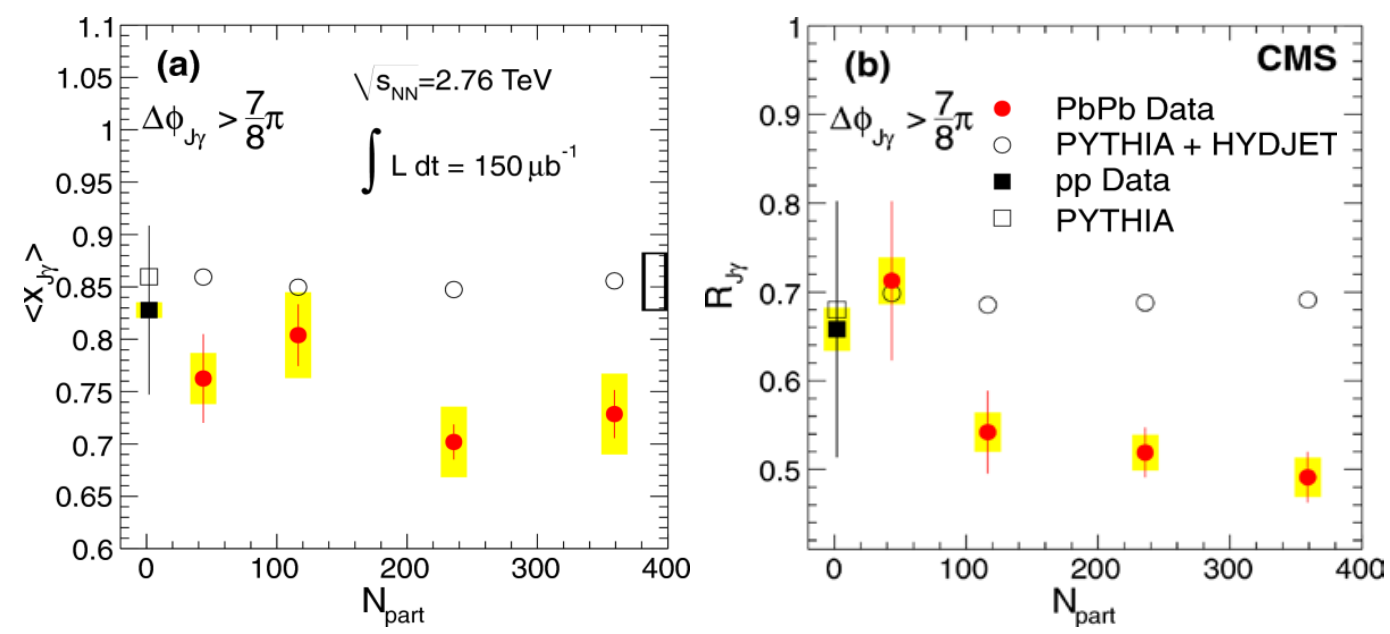

Figure 5: (a) The average ratio of jet-to-photon transverse momentum as a function of the number of participant nucleons. (b) The average fraction of isolated-photons-to-jets as a function of the number of participant nucleons [8].

\section{Conclusion}

In summary, medium effects are clearly evident in both jet+jet and jet + isolated- $\gamma$ events produced in $\sqrt{S_{N N}}=2.76 \mathrm{TeV} \mathrm{PbPb}$ collisions. Such effects show up in momentum and number differences measured between central and peripheral collision centrality. The jet + isolated- $\gamma$ event channel holds great promise for future studies of hadronic energy loss mechanisms. 


\section{References}

[1] F. Karsch and E. Laerman, Thermodynamics and in-medium properties from lattice QCD, in: R. Hwa (Ed. ), Quark-Gluon Plasma III, World Scientific, Singapore, (2004), 1.

[2] CMS Collaboration, The CMS experiment at the CERN LHC, JINST 0803 (2008) S08004.

[3] J. Casalderrey-Solana and C.A. Salgado, Introductory lectures on jet quenching in heavy ion collisions, Acta Phys. Polonica B 38, (2007) 3731.

[4] Zaida Conesa del Valle, Vector bosons in heavy-ion collisions at the LHC, Eur. Phys. J. C61 (2009) 729.

[5] CMS Collaboration, Study of $Z$ boson production in $\mathrm{Pb}$ - $\mathrm{Pb}$ collisions at $\sqrt{S_{N N}}=2.76 \mathrm{TeV}$. Phys. Rev. Lett. 106 (2011) 212301.

[6] CMS Collaboration, Observation and Studies of jet quenching in $P b$ - $P b$ collisions at $\sqrt{S_{N N}}=$ 2.76 TeV. Phys. Rev C84 (2011) 024906.

[7] CMS Collaboration, Jet momentum dependence of jet quenching in $\mathrm{Pb}-\mathrm{Pb}$ collision as $\sqrt{\mathrm{S}_{\mathrm{NN}}}=$ 2.76 TeV, Phys. Lett. B712 (2012)176.

[8] CMS Collaboration, Studies of jet quenching using isolated-photon + jet correlations in $\mathrm{Pb}-\mathrm{Pb}$ and p-p collisions at $\sqrt{\mathrm{S}_{\mathrm{NN}}}=2.76 \mathrm{TeV}$, Phys. Lett. B712 (2012) http://dx.doi.org/10.1016/j.physletb.2012.11.003 